\title{
Long-term Effects of Hydroxyurea in Psoriasis
}

\author{
M. G. C. DAHL, J. S. COMAISH
}

British Medical fournal, 1972, 4, 585-587

\section{Summary}

Hydroxyurea is an effective treatment for psoriasis but consistently produces macrocytosis in peripheral blood with a fall in haemoglobin levels and white cell counts. In this long-term study of 16 patients anaemia and leukopenia have proved frequent and troublesome side effects requiring discontinuance of treatment in many cases. In view of these findings hydroxyurea cannot be recommended as the drug of first choice even for treating severe and intractable psoriasis, and it is our opinion that methotrexate is safer and at least as effective in these circumstances. The clearest indications for the use of hydroxyurea appear to be in life-ruining psoriasis when methotrexate treatment has either failed or is contraindicated because of liver damage. A great deal more investigative work is required before these indications can be generally extended.

\section{Introduction}

Most patients with psoriasis can be adequately controlled with topical applications, though the effective use of these may require occasional periods of treatment in hospital. A small proportion of the most severely affected patients, however, are resistant to all efforts with topical therapy, while others respond in hospital only to relapse shortly after discharge. Many such patients in recent years have been treated with antimetabolic drugs, particularly methotrexate, but recent work has emphasized the hazards of long-term methotrexate (Baker, 1970; Dahl et al., 1971) and indicates the desirability of exploring alternative systemic treatment for prolonged use.

Hydroxyurea, an inhibitor of deoxyribonucleic acid (DNA) synthesis, has recently been reported to be effective in the shortterm treatment of psoriasis (Yarbro, 1969; Leavell and Yarbro, 1970). We report here our experience in treating 16 severely affected psoriatic patients with hydroxyurea continuously for up to 30 weeks. The purpose of the study was to assess the value of the drug in a group of severely affected and resistant psoriatic patients, and to study the problems of toxicity with long-term treatment.

\section{Patients and Methods}

Eleven men and 5 women were studied (age range 30-71 years, mean 56). All suffered from disabling psoriasis resistant to dithranol, coal tar, and corticosteroid preparations. Twelve patients had been previously treated with methotrexate and two with systemic corticosteroids. Topical therapy was not altered either at the commencement of or during treatment with hydroxyurea.

Four patients were receiving methotrexate at the beginning of the study. This was discontinued in all of them within 2-3 weeks of starting hydroxyurea. Three of these patients had previously shown severe relapse within $2-3$ weeks of metho-

University Department of Dermatology, Royal Victoria Infirmary, Newcastle upon Tyne

M. G. C. DAHL, M.B., M.R.C.P., M.R.C. Research Fellow

J. S. COMAISH, M.B., M.R.C.P., Consultant Dermatologist trexate withdrawal, and since all three relapsed during the change-over period assessment of their response to hydroxyurea was possible. The other patient had inoperable colonic carcinoma and received hydroxyurea for only six weeks. His response to treatment was assessed on the basis of relapse after withdrawal of hydroxyurea and his response to a further course of the drug. One patient was taking prednisone $7.5 \mathrm{mg}$ daily, and continued to do so throughout the treatment period.

Liver biopsy material was available from 10 of the patients who had been previously treated with methotrexate. One patient had established cirrhosis accompanied by ascites and splenomegaly, three had biopsy evidence of hepatic fibrosis, and the others showed non-specific fatty change and round cell infiltration. A further patient who had never received methotrexate had a persistently raised serum alkaline phosphatase. His liver biopsy showed mild non-specific hepatitis. One patient had mild acromegaly, and was taking methyldopa for hypertension. With these exceptions, and that of the patient with carcinoma, the patients were in good general health and not on other long-term medication.

Before starting treatment the following laboratory investigations were performed: haemoglobin, white cell count, blood urea, serum bilirubin, and alkaline phosphatase and aspartate aminotransferase. Creatinine clearance was estimated in 10 patients. Hydroxyurea was given in an initial dose of $0.5-1.0 \mathrm{~g}$ daily and increased to $1.0 \mathrm{~g}$ daily in all patients after one to three weeks. In eight patients who responded inadequately the dose was increased to $1.5 \mathrm{~g}$ daily. The duration of continuous treatment was from 6 to 28 weeks. Patients were reviewed every one to two weeks initially, and later at intervals not exceeding four weeks. Haemoglobin, white cell count, urea, bilirubin, alkaline phosphatase, and aspartate aminotransferase were estimated at each visit.

The response of the skin to treatment was graded as follows. Excellent-complete to near complete clearing of all lesions. Good-appreciable lessening of extent of lesions or scaling or both, leading to a clear improvement in the patient's social or working life. Fair-slight objective improvement but with little effect on the patient's working or social life.

\section{Results}

RESPONSE OF SKIN LESIONS

Hydroxyurea had a beneficial effect on the skin lesions of all patients treated (see Table). In seven patients the response was classified as excellent, in seven good, and in two fair. First signs of improvement were usually noticed within two to three weeks of starting treatment and consisted of paling of the lesions and a reduction in scaling, together with cessation of itching or soreness. Maximum benefit at any one dose level was achieved after four to eight weeks' treatment.

The response of the four patients who were being treated with methotrexate at the start of hydroxyurea therapy was more difficult to evaluate. Three showed relapse of their psoriasis during the transition period, however, which was subsequently controlled with hydroxyurea thus indicating the drug's effectiveness. The fourth patient (Case 16) remained substantially clear during six weeks of hydroxyurea therapy but relapsed two weeks after discontinuing treatment. On the basis of improvement with a subsequent course of hydroxyurea his response to treatment was classified as good. Of the four patients who were directly changed to treatment with hydroxyurea from metho- 
Clinical Details of Patients

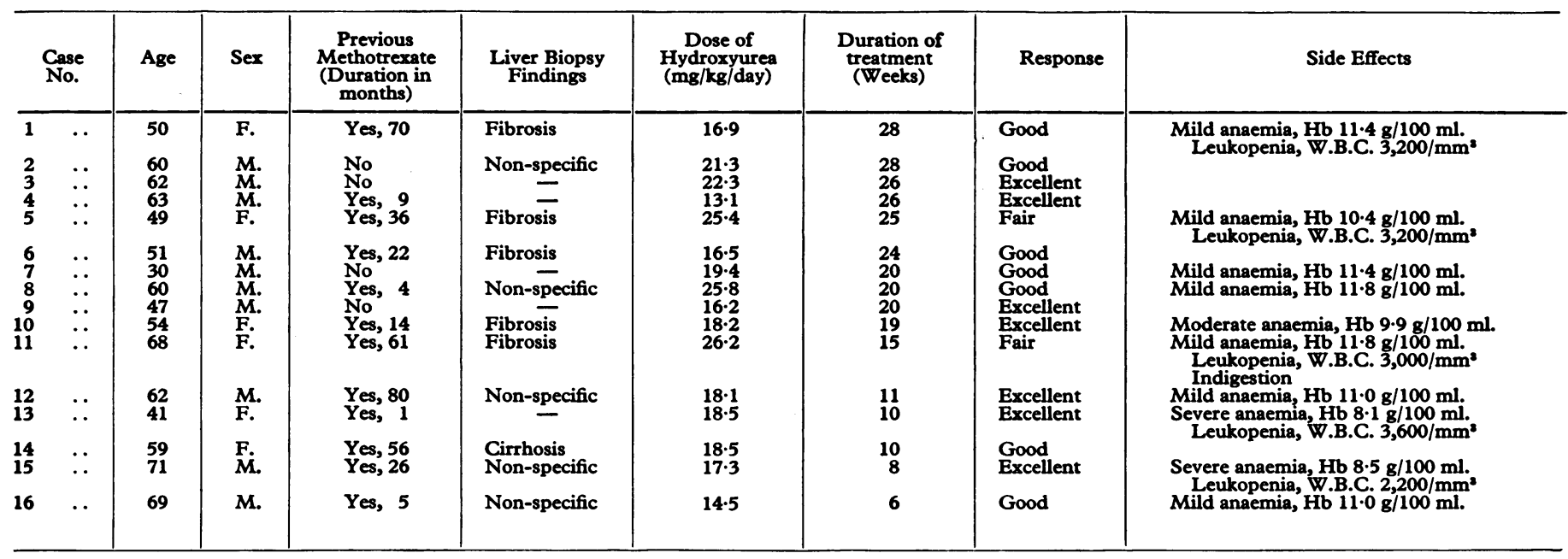

trexate the effect of hydroxyurea was regarded as superior in one, equivalent in two, and clearly less effective in one.

Treatment with hydroxyurea was discontinued in five patients mainly because of anaemia. All five showed relapse and extension of skin lesions within one to four weeks of stopping treatment.

\section{TOXICITY}

Evidence of impaired bone marrow function was seen in all patients. A fall in haemoglobin concentration or reduction in white cell count or both was most evident during the first few weeks of treatment. The effect of seven to nine weeks' treatment with hydroxyurea is shown in Fig. 1. Haemoglobin values fell from a pretreatment mean of $14 \cdot 1 \pm 1 \cdot 2$ (S.E.) $\mathrm{g} / 100 \mathrm{ml}$ to

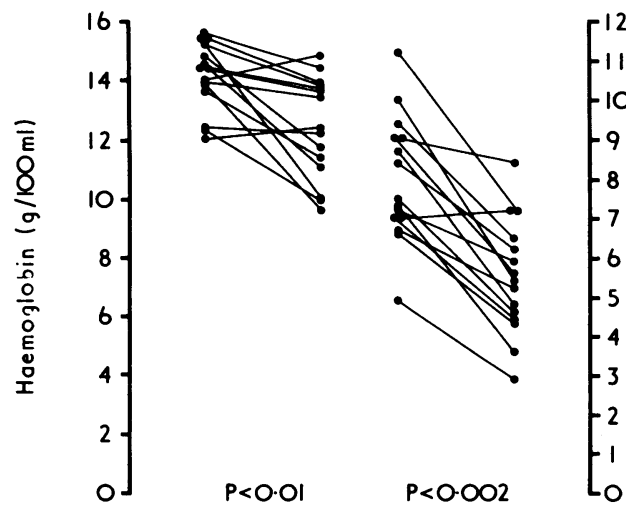

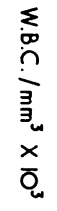

FIG. 1-Haemoglobin and W.B.C. before and after 7-9 weeks' treatment with hydroxyurea $1.0-1.5 \mathrm{~g}$ daily.

$12.5 \pm 1.7 \mathrm{~g} / 100 \mathrm{ml}(\mathrm{P}>0.01)$ and the white cell counts fell from a mean of $8,040 \pm 1,680 / \mathrm{mm}^{3}$ to $5,480 \pm 1,470 / \mathrm{mm}^{3}(P>0.002)$. In 10 patients the haemoglobin fell to below $12.0 \mathrm{~g} / 100 \mathrm{ml}$ and in two a fall to levels of 10.0 and $8.1 \mathrm{~g} / 100 \mathrm{ml}$ led to cessation of treatment after 9 and 10 weeks' treatment respectively. In four other patients mild to moderate anaemia contributed substantially to the decision to discontinue treatment. The anaemia was typically normochromic with anisocytosis but some macrocytosis was seen in all patients.

Leukopenia (W.B.C. $<4,000 / \mathrm{mm}^{3}$ ) was found at some time in five patients. This was accompanied in all cases by some degree of anaemia $(\mathrm{Hb}<12.0 \mathrm{~g} / 100 \mathrm{ml})$. As with haemoglobin levels the white cell counts tended to level out after a relatively steep fall during the first few weeks of treatment, but in several patients levels of $3,500-4,500 / \mathrm{mm}^{3}$ persisted during treatment (Figs. 2 and 3). The fall in circulating white cells involved predominantly the numbers of neutrophil polymorphs and to a lesser extent the circulating lymphocytes. Platelet counts were not routinely made but were usually assessed as "normal in quantity" on the blood film appearance. The lowest recorded platelet count was $100,000 / \mathrm{mm}^{3}$, and there were no clinical problems related to thrombocytopenia.

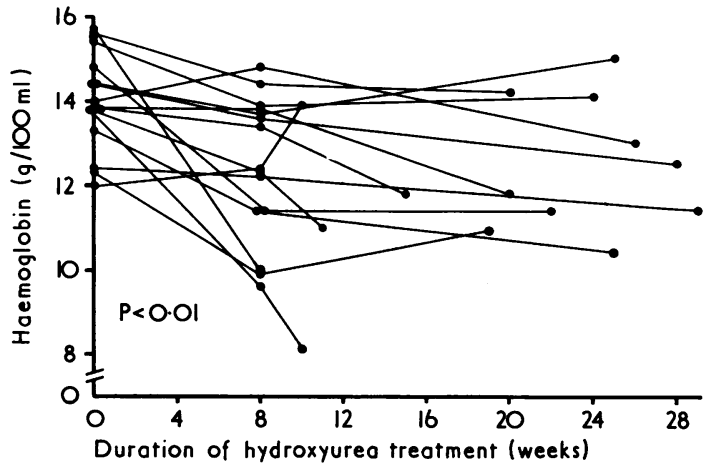

FIG. 2-Haemoglobin levels in patients treated with hydroxyurea for eight weeks or longer. Case 16 not included.

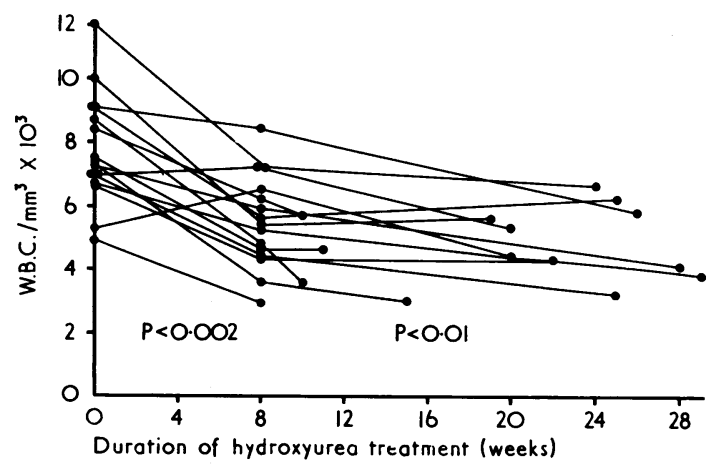

FIG. 3-W.B.C. levels in patients treated with hydroxyurea for eight weeks or longer. Case 16 not included.

\section{RENAL FINDINGS}

Creatinine clearance tests were performed in 10 patients before treatment. Normal values $(100-150 \mathrm{ml} / \mathrm{min})$ were found in six patients, while four showed a mild reduction in clearance 
(54-80 $\mathrm{ml} / \mathrm{min})$. Levels of blood urea and serum creatinine remained entirely normal during treatment.

\section{HEPATIC FUNCTION}

No evidence of hepatic dysfunction attributable to hydroxyurea was encountered. Twelve patients had previously been treated with methotrexate, and of these five had liver biopsy evidence of hepatic fibrosis and one had frank cirrhosis with ascites before hydroxyurea was started. With one exception liver function tests were normal before hydroxyurea treatment was started and remained normal throughout treatment. This patient (Case 2) had a persistently raised serum alkaline phosphatase of between 98 and 122 IU (normal 20-90) before treatment. Isoenzyme electrophoresis showed this to be hepatic in type. A liver biopsy showed only minor non-specific abnormalities. During treatment the alkaline phosphatase levels remained at or just above the upper limit of normal and no other abnormalities were found. One patient had a repeat liver biopsy which showed minor non-specific changes as before.

\section{OTHER EFFECTS}

Hyperpigmentation was noted in seven patients. In four of these the increased pigment was confined to areas of skin previously affected by psoriasis, which became a uniform yellow-brown or grey-brown in colour. In two patients who had previously had very extensive psoriasis the pigmentation was more generalized. One patient who had had widespread freckling of the skin since childhood showed a remarkable increase in size and darkness of the lesions during hydroxyurea treatment. In general the pigmentation faded after some weeks even if hydroxyurea was continued.

Several patients reported tiredness and a lack of energy. In most cases this could be accounted for by anaemia. One patient complained of indigestion and dysphagia during treatment but was subsequently shown to have an oesophageal stricture. Apart from this, gastrointestinal symptoms were conspicuously absent.

\section{Discussion}

Our results confirm the effectiveness of hydroxyurea in healing psoriatic lesions (Yarbro, 1969; Leavell and Yarbro, 1970; Stein et al., 1971; Rosten, 1971). The rapid relapse after withdrawal of the drug in the present patients was predictable since these subjects were selected because of the severity and longterm intractability of their psoriasis.

The precise mechanism by which hydroxyurea acts in psoriasis is not known, but direct inhibition on DNA synthesis is likely to play a major part in its therapeutic effect. Macrocytes can be found in human peripheral blood within 24 hours of administration of hydroxyurea (Bergsagel, et al., 1964), and the same workers found that a megaloblastic marrow was consistently present within one week of starting treatment. Without exception the patients under study developed macrocytosis during treatment even in the absence of frank anaemia. Values for mean corpuscular volume consistently lie between 110-125 $\mu \mathrm{m}^{3}$ (normal 76-96 $\mu \mathrm{m}^{3}$ ). Thurman and Watkins (1964) showed that the megaloblastosis was not accompanied by changes in serum levels of vitamin $B_{12}$ or folate, and that the changes were not prevented by earlier or concomitant therapy with folic acid and vitamin $B_{12}$. Experimentally the inhibitory effect on DNA synthesis induced by short-term exposure to hydroxyurea has been rapidly reversible. We therefore expected a rapid reticulocyte response with return to a normal blood picture after withdrawal of hydroxyurea but this took up to three weeks in most of the present patients. This prolonged effect is unlikely to be due to persistence of hydroxyurea in the body, since in studies using hydroxyurea- ${ }^{14} \mathrm{C}$ in rats and mice up to $95 \%$ of the administered dose was excreted within 24 hours (Adamson et al., 1965). Moreover, the rapid reversibility of its effect in short-term studies indicates that hydroxyurea is not firmly bound to its site of action as is the case with methotrexate. It is likely that bone marrow stem cells become depleted by direct cytotoxic action (Sinclair, 1967; Phillips et. al., 1969). Similarly, a cytotoxic effect on epidermis (Hennings and Devik, 1971) may be important in the healing of the psoriatic lesion.

Abnormalities of renal function during hydroxyurea therapy were reported by Samuels and Howe (1964). They noted mild albuminuria with an increase in white cells and granular casts and occasionally red cells in the urine in some patients. They also found decreased phenolsulphonphthalein excretion and a rise in serum uric acid levels possibly due to impaired renal tubular function. The dose of hydroxyurea we used (about $20 \mathrm{mg} / \mathrm{kg} /$ day) was $25-50 \%$ of that used by Samuels and Howe. Repeated examination of midstream urine specimens in the present patients failed to show albuminuria or an abnormal deposit of cells or casts except in one patient who had had frequent episodes of urinary tract infection before the start of treatment. Serum creatinine levels remained consistently within normal levels, and clearance values remained essentially unchanged.

Liver toxicity is proving to be one of the most important long-term hazards of methotrexate therapy for psoriasis (Dahl et al., 1971). This effect was the main stimulus to our examining the long-term use of hydroxyurea. We know of only one reported case of hepatic dysfunction during hydroxyurea treatment (Thurman et al., 1963) and no details were given. Many of the patients in the present study had evidence of previous liver damage from methotrexate but so far we have not found any evidence of liver toxicity on the basis of serial liver function tests.

The hyperpigmentation noted in seven of the patients is of interest. Occasionally hyperpigmentation of previously psoriatic skin is seen after treatment with conventional topical therapy or methotrexate. However, all of the patients had had previous treatment with dithranol, coal tar, ultraviolet irradiation, topical corticosteroids, and, in some cases, methotrexate. None had ever noted hyperpigmentation previously. The hyperpigmentation appears to fade gradually, and we had regarded it as postinflammatory in nature. However, we have recently seen a patient, not included in the present study, who developed gross patchy hyperpigmentation, not only in previously involved areas but also in non-involved areas on the thighs and areas such as the scrotum and areolae of the nipples, which are normally more pigmented than other parts. This observation raises the possibility that a central mechanism such as an increase in melanocyte stimulating hormone production might be involved, and this man's serum melanocyte stimulating hormone was found to be $216 \mathrm{pg} / \mathrm{ml}$ (normal 40-100 pg/ml).

\section{References}

Adamson, R. H., Ague, S. L., Hess, S. M., and Davidson, J. D. (1965) Fournal of Pharmacology and Experimental Therapeutics, 150, 322.

Baker, H. (1970). Transactions of the St. Fohn's Hospital Dermatological Society, New Series, 56, 111.

Bergsagel, D. E., Frenkel, E. P., Alfrey, C. P. jun., and Thurman, W. G. (1964). Cancer Chemotherapy Reports, 40, 15.

Dahl, M. G. C., Gregory, M. M., and Scheuer, P. J. (1971). British Medical Fournal, 1, 625.

Hennings, H., and Devik, F. (1971). Cancer Research, 31, 277.

Leavell, U. W., and Yarbro, J. W. (1970). Archives of Dermatology, 102, 144. Phillips, F. S., Sternberg, S. S., and Lenaz, L. (1969) Recent Results in Cancer Research, 17, 104 .

Rosten, M. (1971). British fournal of Dermatology, 85, 177.

Samuels, M. L., and Howe, C. D. (1964). Cancer Chemotherapy Reports, 40, 9. Sinclair, W. K. (1967). Cancer Research, 27, 297.

Stein, K. M., Shelley, W. B., and Weinberg, R. A. (1971). British fournal of Dermatology, 85, 81.

Thurman, W. G., et al. (1963). Cancer Chemotherapy Reports, 29, 103.

Thurman, W. G., and Watkins, W. L. (1964). Cancer Chemotherapy Reports, 40,23 .

Yarbro, J. W. (1969). Lancet, 2, 846. 